\title{
Microsensors for In-situ Chemical, Physical and Radiological Characterization of Mixed Waste (73808)
}

\section{Research Objective:}

Portable, real-time, in-situ chemical, physical, and radiological sensors for the characterization and monitoring of transuranic waste, mixed waste, ground water, contaminated soil, and process streams are needed within the DOE complex. A continuation of this basic research program is proposed to study the influence of control of the electrochemical potential of a metallic coating on a microcantilever as a means of developing specific and highly sensitive sensors. Basic research will be needed to understand the influence of variation of electrochemical potential on the bending of cantilevers in an electrolyte solution. Changes in the chemical potential of a metal-electrolyte interface, affected by changing the applied potential, leads to a change in the depletion or accumulation of substances at the interface. This change in the surface excess at the interface is reflected in a change in the interfacial tension, which is sensitively detected as cantilever deflection. Deposition of electroactive heavy metals as well as the adsorption of metal oxide species will be detectable as a cantilever bending.

We plan to continue field-testing cantilever sensors at DOE sites as appropriate. Practical sensors for $\mathrm{Hg}$ and $\mathrm{CrO}_{4}{ }^{-2}$ have been developed, and the former has been field tested. A sensor for large poorly hydrated anions $\left(\mathrm{ClO}_{4}{ }^{-}, \mathrm{ReO}_{4}{ }^{-}, \mathrm{TcO}_{4}{ }^{-}\right)$based on a quarternary ammonium SAM coating is under development and will be field tested when appropriate. The advantage of cantilever sensors is that once the basic platform is developed, it can be the basis for a plethora of inexpensive, miniature sensors. This program will take advantage of advances in cantilever technology made by other programs as well.

\section{Research Progress and Implications:}

Analysis needs exist today for contaminants of concern across the DOE Complex that broadly include radionuclides, metals, and dense nonaqueous phase liquids (DNAPLs). Specifically, key chemicals by two general groups are heavy metals (lead, chromium(VI), mercury, zinc, beryllium, arsenic, cadmium, and copper) and transuranic elements (to include uranium). Although these two general groups and the members of each group are chemically different, having no general molecular recognition characteristics in common, they have one characteristic in common in the sense that many are electrochemically active. Many of the heavy metals (generally RCRA waste) exist in ground waters or in transuranic and mixed waste as oxidized ionic species (Pb(II), $\mathrm{Cr}(\mathrm{VI}), \mathrm{Hg}(\mathrm{II}), \mathrm{Cd}(\mathrm{II})$, and $\mathrm{Cu}(\mathrm{II})$ ) that can be electroplated on a gold, platinum, or nickel surface. . One method of achieving selectivity that has been exploited to date is by using chemically selective coatings on one surface of the cantilever. Based on the principals of molecular recognition, selective coatings have been developed by this research program for $\mathrm{Cs}^{+}, \mathrm{CrO}_{4}{ }^{2-}, \mathrm{Ca}^{2+}, \mathrm{Cu}(\mathrm{II}), \mathrm{Hg}(\mathrm{II})$, and $\mathrm{CH}_{3} \mathrm{Hg}^{+}$(monomethylmercury). We will continue investigations of sensors based on this general principle. It is our perception that another innovation in microcantilever sensors can come from electrochemical polarization of a metallic coating on one surface of a cantilever. The development of a micromachined cantilever platform, which would allow various metals to be coated on one surface of the cantilever and have the capability for potentiostatic control of both reference and active analysis cantilevers, is one of the goals of this project. An improved understanding of both the equilibrium and temporal behavior of an electrified cantilever will lead to the development of new sensors. 
Electrodeposition of $\mathrm{Pb}, \mathrm{Hg}, \mathrm{Cu}$, and As species have been established at gold and platinum surfaces. These electrochemical reactions have been utilized for analytical purposes for many decades, and the combination of electrochemical reduction and cantilever deflection rather than current measurement has the potential to be utilized to make sensitive cantilever sensor elements. We have recently discovered that a gold coated cantilever can be amalgamated with $\mathrm{Hg}$ vapor, and the $\mathrm{Au}(\mathrm{Hg})$ surface functions in electrochemical reactions the same way a mercury electrode functions in well established polarographic methods of electrochemical analysis. The surface potential of a metallic coating on a cantilever will be controlled and modulated by a potentiostat. The metallic film can also be coated with SAMs to selectively concentrate a species at the surface of the cantilever for oxidation/reduction reactions that result in a change in cantilever deflection with respect to a reference cantilever that is held at constant potential. A new initiative in this project is electrochemical perturbation of the metal-solution interface while monitoring microcantilever deflection, providing a new method of achieving selectivity in cantilever sensors while maintaining high sensitivity. We are studying voltammetry at microcantilever electrodes, and we can measure the first derivative of stress with respect to potential in addition to the current, using these measurements to both identify and quantify analytes.

Surface free energy density can be represented by surface stress in the Suttleworth equation

$$
\sigma=\gamma+\partial \gamma / \partial \varepsilon
$$

where $\sigma$ is the surface stress, $\gamma$ is the surface free energy, and $\varepsilon$ is the elastic surface strain (the latter of which is usually small and can be ignored). The radius of curvature and the adsorptioninduced surface stress on the microcantilever can be related using Stoney's formula with the result that deflection is proportional to surface stress. An understanding of the dependence of surface stress on charge and potential at the solid-electrolyte interface will lead to a better understanding of processes occurring at an electrified interface. The Gibbs-Duhem equation for an interface can be written as

$$
d \gamma=-q d E-\sum_{i} \Gamma_{i} d \mu_{i}+(\sigma-\gamma) d \varepsilon
$$

where $\mathrm{E}$ is the electrode potential, $\mathrm{q}$ is the surface charge, $\Gamma_{\mathrm{i}}$ is the surface excess of species $\mathrm{I}, \mu_{\mathrm{I}}$ is the chemical potential of species I, and the other symbols have been previously defined, and this equation provides a relationship between surface free energy, surface coverage, and stress. This equation can be differentiated with respect to potential to obtain the generalized Lippman equation that allows the derivative of deflection with respect to potential to be related to the surface charge during a deposition or stripping process.

The potential-induced surface stress of a gold-coated cantilever electrode was investigated in an electrochemical cell. Gold-coated atomic force microscope microcantilevers were used as working electrodes to measure the current-potential response (by cyclic voltammetry) and simultaneous bending characteristics in solutions of $\mathrm{NaNO}_{3}$ and $\mathrm{K}_{3} \mathrm{Fe}(\mathrm{CN})_{6} / \mathrm{NaNO}_{3}$. The measured change of differential surface stresses of electrode microcantilever was introduced by electrochemical potential-induced changes in surface charge density, ion adsorption/desorption, and electron transfer across the electrode surface. Our results 
indicated that the differential surface stress of microcantilever were introduced by electrochemically induced changes in charge density due to both ion adsorption/desorption and electron transfer on the electrode surface.

Atomic force microscopy microcantilevers coated with gold were used as the working electrode to monitor the deposition and stripping processes of copper for different concentrations of $\mathrm{Cu}^{2+}$ in a $0.1 \mathrm{M} \mathrm{NaNO}_{3}$ supporting electrolyte $(\mathrm{pH}=4.0)$. These processes, combined with electrochemical polarization techniques, caused microcantilever bending by changing the surface stress of the microcantilever electrode. Under these conditions, measurements of microcantilever surface stress shows high sensitivity for the detection of trace amounts (down to $10^{-11} \mathrm{M}$ ) of $\mathrm{Cu}^{2+}$. Anodic stripping of the copper shows a peak in the first derivative of surface stress with respect to potential. The potential of the peak allows the copper to be identified. This potentialcontrolled method offers the promise of achieving chemical selectivity for microcantilever sensor applications in liquid environments for a variety of analytes while still maintaining high sensitivity

\section{Planned Activities:}

The methods of surface modification, based on principles of molecular recognition, which have been successful in rendering the cantilever selective for certain analytes can also be employed with electrochemical polarization of the surface to achieve addition selectivity. The work is being extended to the analysis of $\mathrm{Pb}$ and to a study of the electrolysis of a complex of $\mathrm{Cu}$ on the surface of the cantilever.

\section{Information Access:}

"Detection of $\mathrm{CrO}_{4}{ }^{2-}$ Using a Hydrogel Swelling Microcantilever Sensor," Yifei Zhang, HaiFeng Ji, Gilbert M. Brown, and Thomas Thundat, Anal Chem, 2003, 75, 4773-4777

"Detection of heavy metal ions using protein-functionalized microcantilever sensors" S. Cherian, R.K. Gupta, B.C. Mullins, and T. Thundat, Biosensors and Bioelectronics, 19, 411- 416 (2003)

"Observation of the Surface Stress Induced in Microcantilevers by Electrochemical Redox Processes,” F. Tian, J.H. Pei, D.L. Hedden, G.M. Brown, T. Thundat, Ultramicroscopy, in press.

"Detection of Copper with Potentiostatically Controlled Microcantilever Sensors, " Gilbert M. Brown, Fang Tian, Jianhong Pei, and Thomas Thundat, Proceedings of the Electrochemical Society, in press.

“A pH Sensor Based on a Microcantilever Coated with Intelligent Hydrogel,” Yifei Zhang, HaiFeng Ji, Dale Snow, Ray Sterling, and Gilbert M. Brown, Instrum. Sci. Tech, 2004 32, 361-369 